\title{
System burning out our doctors - study
}

Three-quarters of doctors, mostly juniors, at Cape Metropole district and community level public health facilities are suffering clinically significant burnout, more than half are emotionally exhausted and $64 \%$ have developed a 'callous or depersonalised' perception of their patients.

These are the findings of a 2010 study by Dr Liezel Rossouw, of the Division of Family Medicine and Primary Care at the University of Stellenbosch, who began her probe after encountering a 'perplexing' attitude towards patients among her colleagues at community health centres. 'I thought this was quite a paradox, since surely we become doctors to help our patients,' she said, admitting that she suspected burnout to be the cause.

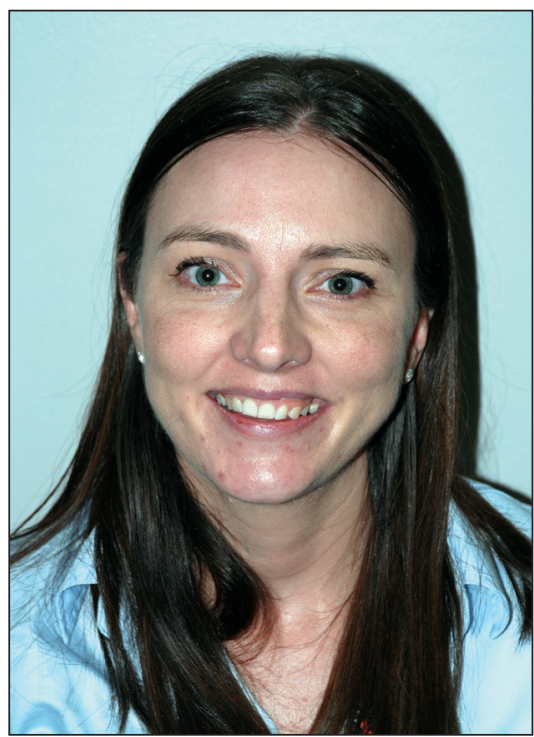

Dr Liezel Rossouw of the Division of Family Medicine and Primary Care at the University of Stellenbosch.

Among the most shocking findings in the study of 132 doctors was that only five per cent felt they were 'engaged' in their work and that the 'burnout' sufferers were using no form of treatment, indicating high levels of denial or stigma. Rossouw singled out community service doctors and medical officers as suffering the greatest stress, with resultant depression and burnout. Their more experienced colleagues displayed higher resilience, described as the most important modifying factor. (Resilience is characterised by past successes, the strengthening effect of stress, the recognition of limits to control, engaging the support of others, an actionoriented approach, a tolerance of 'the negative effect', adaptability, optimism, faith and a sense of humour.)

\section{Address systemic faults - or keep} shedding doctors

It is the first study of its kind in the country and she and her co-authors (an impressive array of the top psychiatry and family medicine lecturers on Rossouw's campus, namely Professors Soraya Seedat, Robin Emsley and Sharain Suliman of the Department of Psychiatry and Dr Dirk Hagemeister of the Division Family Medicine and Primary Care) say addressing the causal factors could have a profound impact on the outward migration of doctors and enhance primary health care delivery across the country. They called for similar studies in other (mostly less well-equipped and worse-managed) metros and provinces to enable comparisons and input to a nationally coordinated intervention. 'These results highlight the urgent need for policy makers to address the work circumstances of these medical practitioners and create a system that would enhance motivation and job satisfaction', the report says. The authors classified burnout as 'a persistent, negative, workrelated state of mind in "normal" individuals that is primarily characterised by exhaustion and is accompanied by distress, a sense of reduced effectiveness, decreased motivation and the development of dysfunctional attitudes and behaviours at work'.

\section{Among the most shocking} findings in the study of 132 doctors was that only five per cent felt they were 'engaged' in their work and that the 'burnout' sufferers were using no form of treatment, indicating high levels of denial or stigma.

Just under three times as many doctors experienced moderate depression when compared with the life-time prevalence of depression documented in the general South African population (27\% versus $9.8 \%$ ) and three per cent suffered severe depression. The number of hours worked ( $84 \%$ worked overtime with $54 \%$ working 64 or more hours of overtime per month), the work load, working conditions and public systemrelated frustrations were ranked as the most important factors contributing to burnout. Those who worked longer hours felt they accomplished less, as demonstrated by lower personal accomplishment scores with decreased productivity after a certain number of hours worked, indicating an urgent need for revised after-hours protocols. The authors found that the longer a doctor was qualified or worked in the primary care setting, the lower the levels of emotional exhaustion and depersonalisation experienced - adding weight to ongoing calls for employing and retaining more seniors to supervise and support their junior colleagues.

'These results highlight the urgent need for policy makers to address the work circumstances of these medical practitioners and create

a system that would enhance motivation and job satisfaction.'

\section{Workshop evokes delegates}

The research was presented at a 'Burnout and Wellness in the Doctor' workshop held at the national congress of the South African Academy of Family Physicians at the River Club in Observatory, Cape Town, on 11 May this year. Rossouw urged participants to initiate support for their younger colleagues given that none of those who took part in her study had returned to use the psychology services offered. Getting management to see that doctor support would increase the quality of patient care was 'crucial'. However, unless this was doctor-driven, little change was likely, she added. Dr Maria Christodoulou, Programme Co-ordinator of Integrative Medicine at Stellenbosch University and a highly respected 'health coach' among her peers, described burnout out as 'cortical hi-jack' - a disconnect between cognition and important feedback from the emotional centres.

'When we burn out, the fire goes out, the passion is gone. We become so disconnected from the energy, fire and vitality that reside in us that we turn into an empty shell operated by cognitive function. The way to get back on line is to get back in touch with the internal, to rediscover our passion and realign with the values that brought us to the profession in the first place.'

Christodoulou, who took a year off from her private practice after suffering burnout, said she had learnt a lot about the complexity of managing depression and 'the different ways it shows up and the different contexts more likely to invoke burnout. I also know from coaching people that solutions and strategies for burnout are unique to the individual. Defining what that looks like for each person might be the most important thing.'

Dr Rafiq Lockhat, a clinical psychologist in private practice and former senior lecturer at 


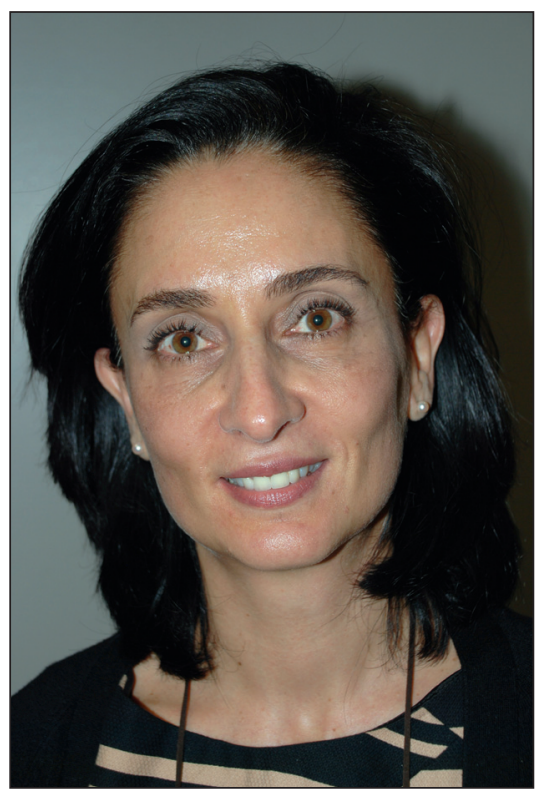

Dr Maria Christodoulou, Programme Coordinator of Integrative Medicine at Stellenbosch University.

the University of Cape Town, warned that 'the collective' would not fix the problem. 'It starts with setting (personal) boundaries.' More senior healthcare workers needed to lose 'that almost sadistic, punishing mentality, where the predominant thought is "I went through that. If I suffered why must you have an easier time?" We need to overcome obstacles to reach the goal of engagement instead of just coping and trying to survive.'

'When we burn out, the fire goes out, the passion is gone. We become so disconnected from the energy, fire and vitality that reside in us that we turn into an empty shell operated by cognitive function.'

\section{Displays of vulnerability rare in doctors}

Christodoulou added: 'We're measured by the number of patients seen, time spent at a desk and patient outcomes from biomedical parameters - where is acknowledgement for the engaged, warm time spent listening, and the care?' One way to change things would be to 'humanise the doctor; to be more honest and vulnerable with one another and our patients. Everyone relates in some way. But we're scared to do that in an environment that measures us by our credentials rather than the quality of care we provide.' To much laughter, she added: 'Have you ever been on a ward round where the consultant talks about the mistakes they made ... discusses all the things that went wrong?' Under any other circumstances, some of the things doctors did within the scope of their practice would be considered criminally insane. 'Sticking knives into people; there's something profound about our license and capacity to do that. We need to teach students the skills to manage the complexity of the emotions our work evokes. We've taught them to shut down. If we could just be present to ourselves and our own emotions whilst functioning professionally ... you can imagine what kind of doctors we'd turn out.' She said research in the corporate world had shown that simply being in a position of power had the capacity to influence the mood and emotional wellbeing of everyone who worked under someone. When that 'superior' walked into a room, within five minutes the mood and emotional wellbeing of everybody present could change (for good or bad). A 'dissonant' leader, not attuned, non-empathic or emotionally absent, caused poor performance and behaviour in an organisation. 'What would it be saying if the dysfunction in our health system is a reflection of our lack of attunement, empathy and presence?' she asked, urging: 'what you say and how you show up and what you tolerate is either contributing to systemic dysfunction or not. What are the values and principles you hold? Define the strategies for making them real in your environment and surround yourself with supportive people. This needs to be doctor-driven. It's not just "they must change things"'. Christodoulou described burnout as 'a very successful intelligent strategy of body and mind demanding that we rediscover our passion'.

Professor Craig Househam, Director General of Health for the Western Cape, lauded Rossouw's work, saying many complaints received by his administration related to the manner in which patients were treated by health professionals, including doctors. A 'significant' investment had been made in an employee health and welfare programme provided by an external service provider whose own findings supported Rossouw's. In a single year (2011/12) over 4600 problems related to provincial health employees were 'managed'. The most frequent included relational, organisational and legal issues and stress, spread across the entire service platform which employed over 29000 people of all categories, mostly from the Metro District Health Services but also from the psychiatric hospitals and Groote Schuur Hospital.

'In each case the employee is offered assistance which may include counselling (either individual or group), coaching or referral', Househam said, adding that serious consideration was being given in the province's
2020 health services strategy to how, with the help of external expertise, the culture of the organisation could be changed and improved.

- It took the deaths of 121 babies after an outbreak of diarrhoeal disease in the poorly served Ukhahlamba District of the Eastern Cape to highlight how disillusioned and unsupported its health care management really was. Managers and staff at various admitting facilities were overwhelmed by a sudden surge of dehydrated babies for nearly four months from January 2008, triggered by terminally decayed township water reticulation and purification systems. Veteran rural public health clinician, Dr Tim Wilson, was sent in by Pretoria to come up with ways to prevent a repeat. He called in Tanya Jacobs, an East London-based social scientist with a Master's degree in Public Health. She tackled the cynicism, demotivation and lack of caring with an interactive workshop. Jacobs reported that the impact of burnout on service delivery included a near-total breakdown in communication, lack of co-operation, demotivated teams, no implementation of decisions, plans or projects, little cohesion or participation, no trust and a 'silo' approach to doing things, plus the concomitant poor productivity. ${ }^{1}$

'What would it be saying if the dysfunction in our health system is a reflection of our lack of attunement, empathy and presence?' she asked, urging: 'what you say and how you show

up and what you tolerate is either contributing to systemic dysfunction or not.'

- Of 22 junior doctor respondents at the Red Cross War Memorial Children's Hospital in 2011, 100\% experienced a high degree of burnout, namely emotional exhaustion, depersonalisation and reduced accomplishment. Of those surveyed $95 \%$ expressed an intention to leave the hospital. The study had a $60 \%$ e-mail questionnaire response rate. ${ }^{2}$

- The 'health and wellness' helpline for Western Cape Metro health staffers is 0800611093.

1. Bateman C. Re-invigorating long-neglected rural health workers. S Afr Med J 2011;101(6):618-620.

2. Stodel JM, Stewart-Smith A. The influence of burnout on skills Stodel JM, Stewart-Smith A. The influence of burnout on skills
retention of junior doctors at Red Cross War Memorial Children's retention of junior doctors at Red Cross War Memorial Ch
Hospital: A case study. S Afr Med J 2011;101(2):115-118.

Chris Bateman

chrisb@hmpg.co.za 